\title{
Projetando a Infraestrutura de Comunicação em Redes Veiculares para a Distribuição de Conteúdos Demandando Níveis Distintos de Desempenho
}

\author{
Cristiano M. Silva ${ }^{1}$, Cristiano G. Pitangui ${ }^{1}$, Alex V. Bastos ${ }^{1}$, Rone I. Silva ${ }^{1}$, \\ Fabrício A. Silva ${ }^{2}$ \\ ${ }^{1}$ Universidade Federal de São João Del-Rei (UFSJ) - Minas Gerais - Brasil \\ ${ }^{2}$ Universidade Federal de Viçosa (UFV) - Minas Gerais - Brasil \\ \{cristiano, pitangui.cristiano, alexvbh, rone\}@ufsj.edu.br \\ fabricio.asilva@ufv.br
}

\begin{abstract}
We investigate the development of Mobile Content Delivery Vehicular Networks. We consider the availability of several content types that must be made available for vehicles at specific locations of the road network. We also assume each content type demanding distinct levels of performance from the network, accordingly to the location where it must be made available. We devise a novel metric for evaluating the performance of vehicular networks, and we use the metric for devising a novel infrastructure deployment strategy. We compare our deployment strategy to locating roadside units at the densest locations of the road network. Results demonstrate our strategy requiring much less roadside units than the baseline to achieve similar levels of performance.
\end{abstract}

Resumo. Este trabalho investiga a distribuição de conteúdos em Redes Veiculares. Considera-se a disponibilidade de vários tipos de conteúdos que devem ser disponibilizados para os veículos em locais específicos da malha viária. Assume-se que cada tipo de conteúdo exige diferentes níveis de desempenho da rede. O trabalho propõe uma nova métrica para avaliar o desempenho de redes veiculares, e utiliza essa métrica para elaborar uma nova estratégia de implantação de infraestrutura para redes veiculares. A estratégia proposta é comparada com o método intuitivo de implantar as unidades de comunicação nos locais de tráfego mais intenso. Os resultados demonstram que a estratégia proposta exige menos unidades de comunicação para atingir a mesma qualidade de serviço.

\section{Introdução}

O rápido crescimento dos centros urbanos aumenta a demanda de transportes para a população, tornando o tráfego num problema central da sociedade moderna. A impossibilidade de alterar constantemente a malha viária motiva os governos a buscarem soluções para maximizar a capacidade das vias já existentes através do desenvolvimento de Sistemas Inteligentes de Transportes (SITs) que se baseiam na comunicação veicular para o gerenciamento da mobilidade urbana. A grande mobilidade dos veículos impõe grandes desafios de comunicação, visto que o canal deve lidar com vários nós (veículos) enviando e recebendo dados simultaneamente. Além disso, diferentemente das redes 
celulares, veículos não exigem cobertura contínua, podendo realizar a troca de dados ao passarem por unidades de comunicação ${ }^{1}$.

Percebe-se que as aplicações veiculares têm evoluído de simples alertas de tráfego a transmissões em tempo real de altíssima qualidade, demandando novas estratégias de gerenciamento das redes de comunicação.Ainda que os veículos possam se comunicar de maneira ad-hoc, o uso de unidades de comunicação alocadas ao longo das vias pode melhorar significativamente o desempenho da rede. Por outro lado, a implantação em larga escala de uma infraestrutura de comunicação pode demandar grandes investimentos [Cheng et al., 2015].

Este trabalho investiga uma rede veicular de distribuição de conteúdos. Já que algumas informações de tráfego são importantes apenas para uma determinada região, assume-se que cada tipo de conteúdo está relacionado a uma região específica da cidade. Além disso, dada a ampla gama de aplicações veiculares vislumbradas, também se considera que cada conteúdo exige diferentes níveis de desempenho. Inicialmente, deriva-se uma métrica para medir a qualidade de serviço oferecida pela infraestrutura suportando a comunicação veicular chamada de Deposição ${ }^{2}$ Sigma $_{(\Sigma)}$. A Deposição Sigma permite que o projetista verifique se uma rede veicular fornece suporte adequado para a entrega de vários tipos de conteúdo, cada um deles exigindo níveis específicos de desempenho da rede, de acordo com a sua localização na cidade.

Em seguida, o trabalho propõe Sigma-g, uma heurística gulosa para resolver a alocação de infraestrutura de forma a se atingir uma Deposição Sigma. Sigma-g é avaliada usando-se o rastro (trace) de mobilidade realístico da cidade de Colônia ${ }^{3}$, Alemanha, composto de 75.515 veículos. Os resultados são comparados com a estratégia intuitiva de se alocar as unidades de comunicação nos locais de maior tráfego, chamada DL (Densest Location). Os resultados demonstram que a heurística proposta reduz de $20 \%-40 \%$ a quantidade de unidades de comunicação preservando a mesma qualidade de serviço.

Diferentemente das abordagens anteriores, a Deposição Sigma é uma métrica orientada a conteúdos para avaliar o desempenho de uma dada deposição de infraestrutura suportando redes veiculares. Este trabalho encontra-se organizado da seguinte forma. A seção 2 apresenta os trabalhos relacionados. A seção 3 apresenta a Deposição Sigma. A seção 4 apresenta uma heurística gulosa para solucionar uma Deposição Sigma. A seção 5 apresenta os experimentos. A seção 6 conclui o trabalho.

\section{Trabalhos Relacionados}

Alguns estudos abordam o problema de distribuição de conteúdo no contexto de redes veiculares [Silva et al., 2014]. Em [Gossa et al., 2008], os autores propõem uma solução de replicação de dados proativa para selecionar veículos como réplicas. Por outro lado, a solução descrita no [Li et al., 2015], replica o conteúdo em unidades de comunicação com base na análise do rastro (trace) de mobilidade real. Da forma

\footnotetext{
${ }^{1}$ Esse trabalho utiliza a expressão "unidades de comunicação" para se referir ao termo "roadside units", cujo significado literal é "unidades de (comuniçação) de beira de estrada".

"O termo "deposição" possui a conotação de implantação ou colocação (de infraestrutura de comunicação para redes veiculares). Assim, uma estratégia de deposição é aquela que identifica onde as unidades de comunicação devem ser implantadas na rede veicular.

${ }^{3}$ Disponível em: http://kolntrace.project.citi-lab.fr/
} 
semelhante, o trabalho [Bruno et al., 2015] investiga um modelo de otimização para replicar os conteúdos nas unidades de comunicação, considerando sua popularidade. O presente trabalho difere das abordagens anteriores no sentido de que os trabalhos anteriores consideram cobertura total da malha viária.

A literatura apresenta várias estratégias para a alocação de unidades de comunicação em redes veiculares. Zheng et al. [2010] apresentam a avaliação da estratégia de implantação considerando a oportunidade de contato. Lee and Kim [2010] propõem uma heurística gulosa para alocar a infraestrutura com o objetivo de melhorar a conectividade entre veículos. Xie et al. [2013] abordam o problema de alocação de unidades de comunicação baseando-se em um modelo probabilístico para inferir as localizações das unidades de comunicação.

Trullols et al. [2010] propõem a modelagem de alocação de unidades de comunicação como um problema de cobertura máxima, assumindo-se conhecimento prévio das trajetórias dos veículos. Essa premissa é relaxada nos trabalhos [Silva et al., 2013, 2015b] através do uso da taxa de migração de veículos entre regiões da área urbana.

De forma geral, o presente trabalho se diferencia da literatura ao propor uma estratégia de implantação que fornece garantia de desempenho mínima especificada em termos da probabilidade de contatos e duração do mesmos, para vários tipos de conteúdo, e de acordo com a localização do mesmo.

\section{Métrica Deposição Sigma}

O planejamento de redes veiculares de distribuição de conteúdos requerem o planejamento da localização física das unidades de comunicação onde se espera que os potenciais clientes passem. Além disso, diferentes tipos de conteúdo podem exigir níveis distintos de desempenho da rede. Assim, este trabalho propõe a Deposição Sigma $(\Sigma)$ como uma estratégia para o planejamento da localização das unidades de comunicação em redes veiculares. Essa estratégia de implantação permite diferentes níveis de disponibilidade de conteúdo ao longo da malha viária.

A principal característica da Deposição Sigma é o fato de considerar as unidades de comunicação armazenando conteúdos diferentes, sendo que cada conteúdo exige níveis distintos de qualidade de serviço especificados através das oportunidades de contatos entre veículos e a infraestrutura, e a duração desses contatos. Uma dada deposição de unidades de comunicação é considerada $\Sigma\left(\begin{array}{c}R_{c}^{3} \\ c\end{array}\right)\left(\begin{array}{c}\rho_{1} \\ \rho_{2}\end{array}\right)$ sempre que $\rho_{2}$ porcento dos veículos que viajam por $R_{c}$ estão conectados a unidades de comunicação tendo o conteúdo $c$ durante (pelo menos) $\rho_{1}$ porcento da duração da viagem ao longo de $R_{c}$, onde $R_{c}$ é uma região contida na malha rodoviária $R$. Os parâmetros $\rho_{1}$ e $\rho_{2}$ indicam garantias de desempenho para veículos encontrando o conteúdo $c$ nas regiões $R_{c}$ da malha rodoviária. Formalmente:

Definição 1 (Deposição: $\Sigma\left(\begin{array}{c}R_{c} \\ c\end{array}\right)\left(\begin{array}{c}\rho_{1} \\ \rho_{2}\end{array}\right)$ ) Assuma que $R_{c} \subseteq R$ represente um subconjunto $d a$ malha rodoviária e que $V$ represente o conjunto de veículos que viaja em $R_{c}$. Considere que $V^{\prime} \subset V$ represente o conjunto de veículos conectados às unidades de comunicação possuindo o conteúdo c durante, ao menos, $\rho_{1}$ porcento da duração da viagem. Uma implantação de unidades de comunicação é considerada $\Sigma\left(\begin{array}{c}R_{c} \\ c\end{array}\right)\left(\begin{array}{c}\rho_{1} \\ \rho_{2}\end{array}\right)$ quando $\frac{\left|V^{\prime}\right|}{|V|} \geq \rho_{2}$.

Nas redes veiculares de distribuição de conteúdos, as unidades de comunicação atuam como servidores que fornecem conteúdo para os veículos. O modelo assume que 
existem diferentes tipos de conteúdos ao longo da rede veicular (tais como informações de trânsito, vídeos, anúncios, etc.). Cada tipo de conteúdo deve estar fisicamente localizado em um subconjunto de células urbanas (regiões da malha rodoviária). Este é um requisito muito importante, pois alguns conteúdos podem ter significado apenas local (por exemplo, informações de trânsito e propagandas não necessariamente precisam ser transmitidos a todos os veículos). Além disso, cada tipo de conteúdo exige níveis distintos de disponibilidade representada em termos da Deposição Sigma, e tais níveis podem variar de acordo com a região (i.e., um subconjunto de células urbanas).

Para resolver a Deposição Sigma, assume-se o particionamento da área urbana em um conjunto de $\psi \times \psi$ células urbanas de mesmo tamanho. As células urbanas podem ter tamanhos arbitrários, a fim de atender às necessidades de projeto da rede. Quando se exige maior/menor precisão, simplesmente aumenta-se/diminui-se o número de células urbanas que cobrem a malha rodoviária. Particionando-se a malha rodoviária, diminui-se o número de locais a serem avaliados para receberem unidades de comunicação. Tal particionamento acarreta, portanto, uma redução do esforço computacional para a obtenção das melhores localidades.

\section{Sigma-g: Uma Heurística para a Deposição de Unidades de Comunicação}

Nesta seção, define-se Sigma-g, uma heurística gulosa para resolver o problema de Deposição Sigma. A heurística considera a malha rodoviária dividida em $\psi \times \psi$ células urbanas. Sem perda de generalidade, este trabalho assume que cada célula urbana é coberta pela implantação de uma única unidade lógica de comunicação. Assim, considera-se que uma unidade lógica é capaz de cobrir completamente uma célula urbana.

Sigma-g recebe como entrada: (a) o subconjunto de células urbanas onde a implantação deve ocorrer $(M)$; (b) o conjunto de veículos viajando por $M(V)$; (c) a trajetória de cada veículo (T); e, (d) os parâmetros de desempenho $\rho_{1}$ e $\rho_{2}$, onde $\rho_{1}$ indica a duração de contato V2I, enquanto o $\rho_{2}$ indica a fração de veículos.

Nota-se que o algoritmo 1 não recebe o tipo de conteúdo como entrada. Assim, deve-se executar Sigma-g para cada tupla <tipo de conteúdo, localização do conteúdo >. A localização do conteúdo é dada por $M$, enquanto que o desempenho requerido para o conteúdo é dado por $\left(\rho_{1}, \rho_{2}\right)$. O conjunto final de unidades de comunicação é simplesmente a união dos conjuntos de unidades retornados por Sigma-g para cada tupla $<$ tipo de conteúdo, localização do conteúdo, $\rho_{1}, \rho_{2}>$.

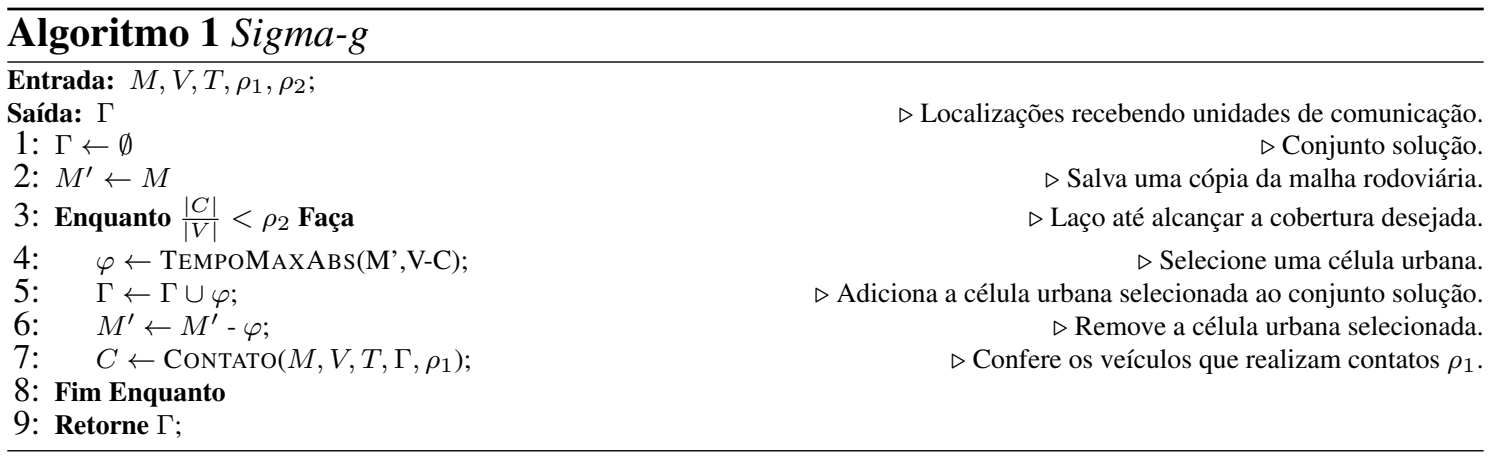

Sigma-g seleciona células urbanas do subconjunto $(M)$ e as coloca no conjunto solução $(\Gamma)$. Cada $M i j$ indica o número de veículos que atravessam a célula urbana $(i, j)$. 
A escolha gulosa baseia-se no tempo de contato absoluto que é fornecido por cada célula urbana ao receber uma unidade de comunicação. Sigma-g calcula um índice para cada célula urbana. Este valor é a soma do tempo de contato V2I total obtido pela alocação de uma unidade na célula. Para calcular este valor, são considerados todos os veículos que satisfizeram o critério $\rho_{1}$ (duração da conexão V2I).

A primeira unidade de comunicação é alocada na célula urbana que possui maior tráfego. A célula urbana selecionada é removida do conjunto de candidatos $\left(M^{\prime}\right)$, e o algoritmo armazena em $C$ os veículos que realizam conexão durante $\rho_{1}$ por cento da duração da viagem. Tal computação é realizada pela função Contato(). A função Contato() usa o subconjunto original de células urbanas $(M)$, o conjunto de veículos $(V)$, a informação de trajetórias $(T)$, as células urbanas recebendo unidades $(\Gamma)$, e $\rho_{1}$. Enquanto a fração de $\rho_{2}$ veículos cobertos não for alcançada, a heurística seleciona de forma iterativa a célula urbana que apresenta a maior contribuição em termos de tempo de contato absoluto para os veículos (ainda) descobertos, usando a função TempoMaxAbs().

Sigma-g possui uma propriedade importante: ela permite que se desconsidere o tipo de conteúdo. O conjunto solução fornecido pela Sigma-g é incremental. Assuma duas deposições: $\Sigma$ ' e $\Sigma$ '. Considere que a implantação $\Sigma^{\prime}$ apresente garantias de desempenho inferiores à implantação $\Sigma$ '. Assim, o conjunto de solução de $\Sigma$ ' demanda menos unidades de comunicação que o conjunto solução de $\Sigma$ ". No entanto, Sigma-g garante que a solução $\Sigma$ ' é um subconjunto de $\Sigma$ '. Assim, $\Gamma^{\prime} \subset \Gamma^{\prime \prime}$.

\section{Experimentos}

Este trabalho compara Sigma-g com DL, uma estratégia intuitiva que aloca as unidades de comunicação nos locais de maior tráfego da malha viária. DL funciona da seguinte forma: enquanto não se atinge a fração de $\rho_{2}$ veículos cobertos durante $\rho_{1}$ porcento da viagem, DL permanece alocando unidades de comunicação, iniciando a partir do local de maior tráfego de veículos.

Os experimentos consideram o rastro (trace) realístico de mobilidade da cidade de Colônia (Alemanha), que é particionada em uma grade ( grid) de $100 \times 100$ células urbanas a fim de se obter células capazes de serem cobertas por apenas uma unidade de comunicação. Tal particionamento gera células urbanas de $270 \mathrm{~m}$ x $260 \mathrm{~m}$, um raio típico de cobertura para a comunicação veicular [Teixeira et al., 2014].

Ao se analisar a qualidade de uma deposição de unidades de comunicação, duas medidas requerem maior atenção: a) investimentos; b) cobertura. Os investimentos são estimados através da análise do número de unidades lógicas requeridas por cada estratégia de deposição, enquanto a cobertura é analisada utilizando-se a Deposição Sigma.

\subsection{Análise de Investimentos: Número de Unidades de Comunicação}

Para estimar os custos financeiros para a criação da rede, a figura 1(a) apresenta a área necessária a ser coberta para que cada estratégia de deposição atinja vários níveis de desempenho especificados por $\left\{\rho_{1}, \rho_{2}\right\}$. O eixo $x$ indica $\rho_{2}$ (fração de veículos), enquanto que o eixo $y$ indica a quantidade de unidades de comunicação que são implantadas por cada estratégia de forma a se obter as Deposições Sigma indicadas no grafico. São plotados cinco conjuntos de dados $\rho_{1}(\{0.1,0.3,0.5,0.7,0.9\})$. 


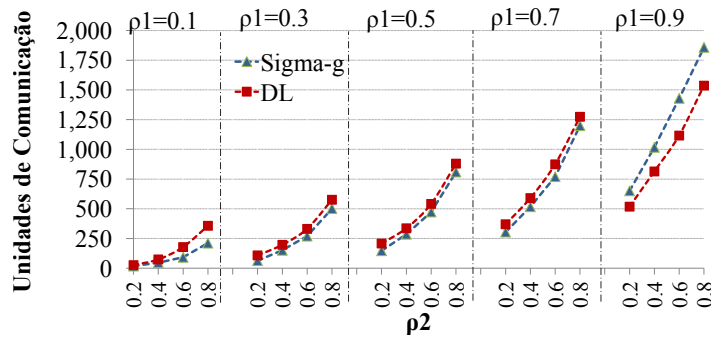

(a) Unidades de Comunicação.

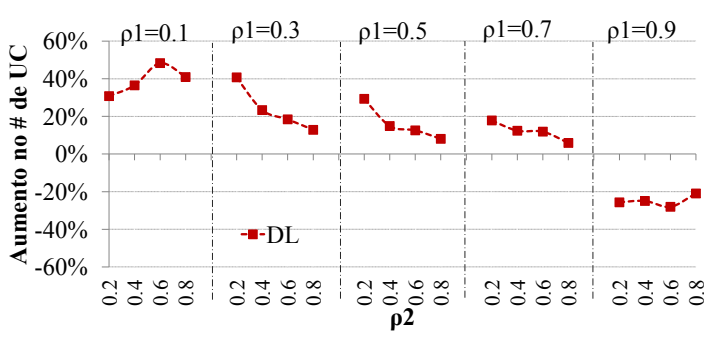

(b) Aumento nas Unidades de Comunicação.

Figura 1. A fig. (a) apresenta a quantidade de unidades de comunicação demandadas por cada estratégia. A fig. (b) apresenta a variação no número de unidades de comunicação demandadas por DL em termos de Sigma-g.

A quantidade de unidades de comunicação demandadas por Sigma-g é indicada na cor azul, enquanto que DL é indicado na cor vermelha. A figura demonstra que Sigma-g demanda menos unidades que DL para todas as configurações, exceto quando $\rho_{1}=0,9$. No entanto, por razões de escala, o gráfico não apresenta com clareza quantas unidades de comunicação Sigma-g economiza em relação à DL.

Com esse intuito, apresenta-se a figura 1(b). Essa figura apresenta a proporção de unidades de comunicação que DL demanda a mais que Sigma-g. Essa proporção varia conforme os valores $\left\{\rho_{1}, \rho_{2}\right\}$. Para $\rho_{1}=0,1$, DL demanda de $25 \%$ até $50 \%$ mais unidades de comunicação que Sigma-g. Conforme aumenta-se $\rho_{1}$ (percentual da viagem que veículos devem ficar em contato com unidades de comunicação), essa relação diminui progressivamente. Para $\rho_{1}=0,9$ DL utiliza menos unidades de comunicação que Sigma-g. Tal nível de desempenho exige uma implantação massiva de unidades de comunicação e a estratégia de cobrir os locais de maior tráfego se mostra bastante útil.

De forma geral, DL apresenta uma curva decrescente para cada conjunto de dados $\rho_{1}$. Aumentando-se $\rho_{2}$, aumenta-se o número de veículos que devem satisfazer o critério de duração de conectividade $\left(\rho_{1}\right)$, o que exige um aumento no número de células urbanas cobertas. À medida que se cobre um maior número de células urbanas, diminuem-se as possíveis combinações de disposições das unidades de comunicação, tornando o problema mais fácil de ser resolvido.

Em última análise, ao se especificar que $100 \%$ dos veículos realizem contatos durante $100 \%$ do tempo de duração da viagem, i.e., uma Deposição $\Sigma\left(\begin{array}{c}R_{c} \\ c\end{array}\right)\left(\begin{array}{c}1.0 \\ 1.0\end{array}\right)$, cada célula urbana que apresente um nível de tráfego maior que zero irá receber uma unidade de comunicação, e ambas as estratégias irão implantar 4.219 unidades lógicas para cobrir todas as células urbanas que apresentam tráfego ${ }^{4}$, e não haverá diferença entre as estratégias.

\subsection{Relacionando Investimentos e Cobertura}

Visto que as estratégias Sigma-g e DL incorrem na implantação de quantidades diferentes de unidades de comunicação, esse experimento caracteriza como a variação no número de unidades de comunicação impacta a cobertura atingida por cada estratégia. Na figura 2(a), a linha azul representa o aumento percentual no tempo de contato (V2I) alcançado por

\footnotetext{
${ }^{4}$ Os traços de Colônia possuem 4.219 células urbanas com tráfego.
} 
DL quando comparado a Sigma-g. Quando a coordenada $y$ é maior do que zero, DL proporciona mais tempo de contato que Sigma-g. Pode-se notar que DL supera em $11 \%$ o tempo de contato fornecido por Sigma-g para o $\Sigma\left(\begin{array}{c}R_{c} \\ c\end{array}\right)\left(\begin{array}{c}0.1 \\ 0.3\end{array}\right)$ e em $32 \%$ para $\Sigma\left(\begin{array}{c}R_{c} \\ c\end{array}\right)\left(\begin{array}{c}0.9 \\ 0.3\end{array}\right)$.

No entanto, deve-se lembrar que DL requer mais unidades de comunicação que Sigma-g. Assim, para uma comparação justa, indica-se também o aumento no número de unidades de comunicação para DL em relação a Sigma-g (linha vermelha). Enquanto DL aumenta em $11 \%$ o tempo de contato de $\Sigma\left(\begin{array}{c}R_{c} \\ c\end{array}\right)\left(\begin{array}{l}0.1 \\ 0.3\end{array}\right)$, ela exige $38 \%$ mais unidades de comunicação que Sigma-g. De forma similar, quando se considera a Deposição $\Sigma\left(\begin{array}{c}R_{c} \\ c\end{array}\right)\left(\begin{array}{l}0.9 \\ 0.3\end{array}\right)$, DL exige $+18 \%$ mais unidades de comunicação que Sigma-g, mas reduz em $3 \%$ o tempo de contato. De forma geral, Sigma-g apresenta um melhor custo-benefício entre investimentos e serviço ofertado.

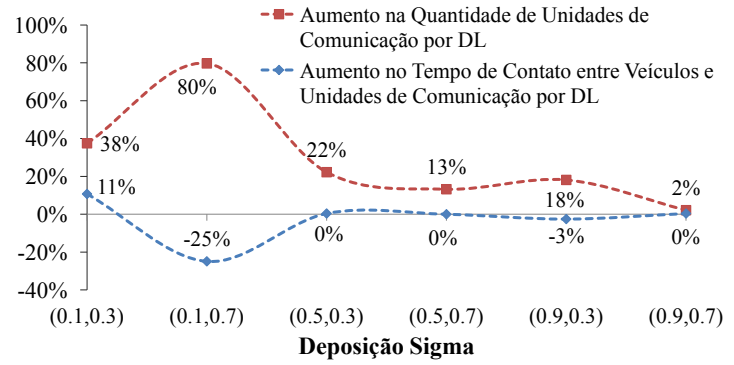

(a) Tempo de Contato V2I.

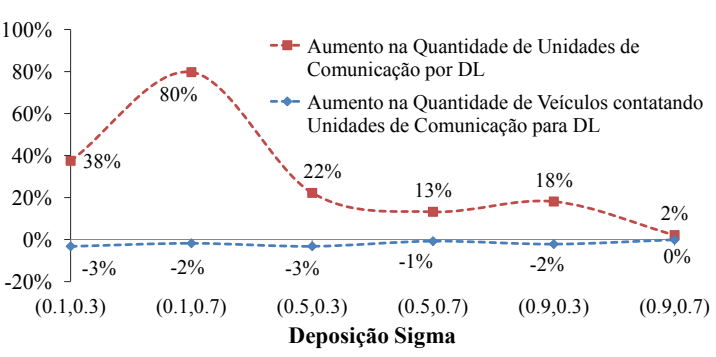

(b) Quantidade de Contatos V2I.

Figura 2. Distribuição de tempo "entre-contatos" para algumas Deposições Sigma. A fig. (a) apresenta a variação percentual no tempo de contato V2I de DL em relação à Sigma-g. A fig. (b) apresenta a variação na quantidade de contatos de $\mathrm{DL}$ em relação à Sigma-g.

Na figura 2(b), a linha azul indica o aumento no número de contatos V2I, em termos percentuais, de DL comparado com Sigma-g. Nota-se um desempenho similar entre as estratégias, com Sigma-g proporcionando um pouco mais de contatos V2I. Mais uma vez, a linha vermelha indica o aumento percentual do número de unidades de comunicação requerido por DL em relação a Sigma-g. É fácil perceber que o aumento no número de unidades de comunicação demandado por DL não retorna em termos de mais veículos contatando unidades de comunicação.

\subsection{Intervalo "Entre-Contatos" do Veículo com Unidades de Comunicação}

O intervalo "entre-contatos" mede o tempo entre duas conexões consecutivas dos veículos a unidades de comunicação. As figuras 3(a)-(b) apresentam o intervalo entre-contatos para Sigma-g e DL considerando diferentes parâmetros Sigma. Para ser justo, calcula-se o tempo entre-contatos em relação à duração da viagem para cada veículo (percentual do tempo de duração da viagem), e a figura apresenta a fração de veículos com um determinado intervalo de tempo entre-contatos (indicado na legenda).

Para melhor entendimento da figura, observe a primeira barra. Ela representa a distribuição dos tempos entre-contatos V2I considerando a Deposição $\Sigma\left(\begin{array}{c}R_{c} \\ c\end{array}\right)\left(\begin{array}{c}0.9 \\ 0.7\end{array}\right)$. As cores representam intervalos entre-contatos. A cor verde indica um tempo entre-contatos muito baixo (0\%-10\% da duração da viagem). Azul indica um tempo entre-contatos médio (20\%-30\% da duração da viagem). Amarelo indica alto tempo entre-contatos 


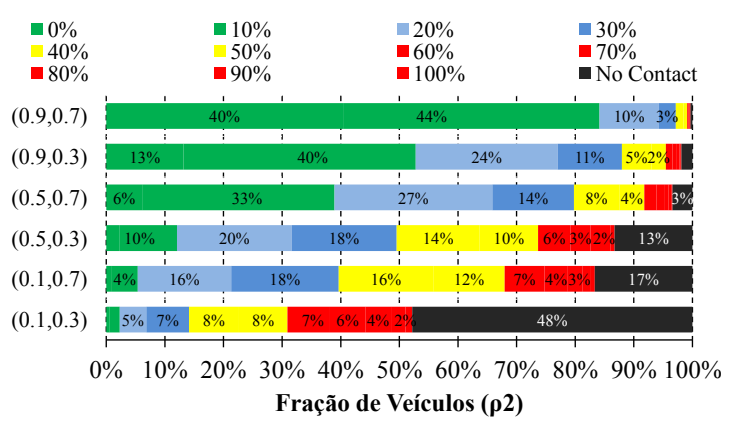

(a) Sigma-g:

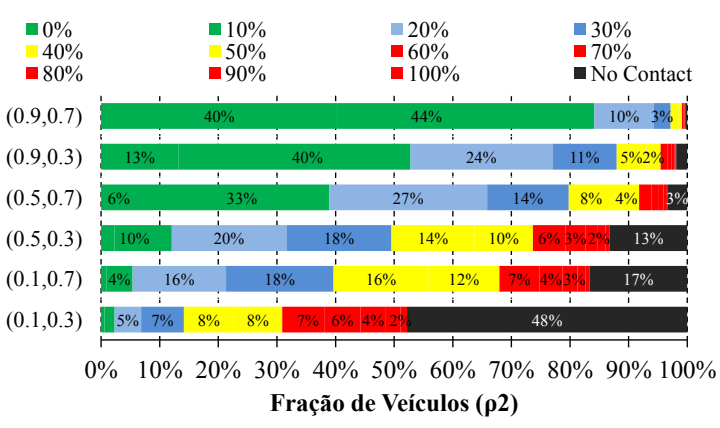

(b) DL.

Figura 3. Distribuição de tempo "entre-contatos" para algumas deposições Sigma. A figura (a) apresenta Sigma-g. A figura (b) apresenta DL.

(40\%-50\% da duração da viagem). Vermelho indica tempo entre-contatos muito elevado ( $>70 \%$ da duração da viagem), enquanto que a cor preta indica a fração de veículos que não realizam contatos com unidades de comunicação durante sua viagem. À medida que se aumentam os níveis de desempenho da implantação, aumenta-se a fração de veículos que apresentam baixos tempos entre-contatos.

\subsection{Análise de Cobertura}

Sempre que se resolve a Deposição $\Sigma\left(\begin{array}{c}R_{c} \\ c\end{array}\right)\left(\begin{array}{c}\rho_{1} \\ \rho_{2}\end{array}\right)$ utilizando alguma estratégia, tem-se, como saída, o número de unidades de comunicação necessário (e suas localizações) na malha rodoviária. Essa disposição de unidades de comunicação permite que $\rho_{2}$ por cento dos veículos realizem contatos V2I durante $\rho_{1}$ por cento da duração da viagem. Ao se implantar as unidades de comunicação e realizar o rastro (trace) de mobilidade, pode-se traçar a relação $\rho_{1} \mathrm{x} \rho_{2}$ (chamada de Rede de Comunicação Delta [Silva et al., 2015a]). Ao se traçar a Rede Delta, caracteriza-se toda a gama de operação da rede, permitindo que o projetista possa medir e comparar o desempenho das redes veiculares.

A heurística Sigma-g é apenas uma estratégia possível para resolver a Deposição $\Sigma\left(\begin{array}{c}R_{c} \\ c\end{array}\right)\left(\begin{array}{c}\rho_{1} \\ \rho_{2}\end{array}\right)$. Já que estratégias distintas tendem a selecionar células urbanas distintas para receber as unidades de comunicação, pode-se inferir que existem diferenças de desempenho da rede como um todo. Por exemplo, uma dada solução para a Deposição Sigma pode permitir mais oportunidades de contato para veículos que viajam distâncias menores, enquanto outras podem beneficiar veículos em viagens mais longas.

Como exemplo, a figura 4(a) plota a Rede Delta para várias Deposições (na legenda). O eixo $x$ indica a duração do contato $\left(\rho_{1}\right)$, enquanto o eixo $y$ indica a porcentagem de veículos $\left(\rho_{2}\right)$. Cada ponto indica a porcentagem de veículos (eixo y) tendo contatos V2I durante (pelo menos) $\rho_{1}$ por cento da duração da viagem (eixo $x$ ). Pode-se notar que à medida que aumentam-se os parâmetros de desempenho $\left(\rho_{1}\right.$ e $\left.\rho_{2}\right)$, também aumenta-se o desempenho da rede (cada Sigma representa uma rede) ao longo de toda a curva, já que utiliza-se uma maior quantidade de unidades de comunicação que fornecem suporte à rede veicular.

Na figura 4(b) usa-se a Rede Delta para comparar Sigma-g e DL. No entanto, em vez de plotar uma curva para Sigma-g, e outra para a DL, plota-se apenas uma curva que representa as diferenças entre ambas as estratégias (a Rede Delta Diferencial). Para cada 


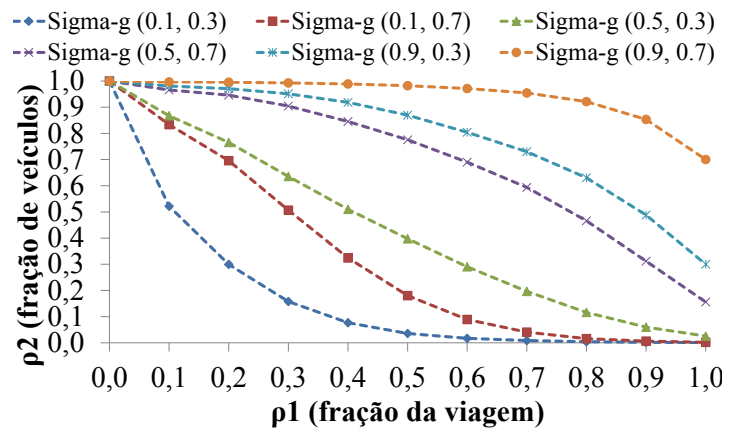

(a) Rede Delta para Sigma-g.

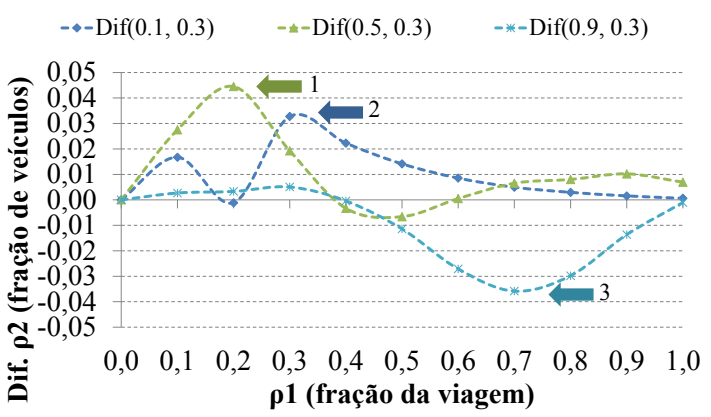

(b) Rede Delta Diferencial (Sigma-g - DL).

Figura 4. A fig. (a) apresenta a relação entre $\rho_{1}$ e $\rho_{2}$ ao se resolver a Deposição Sigma usando-se Sigma-g. A fig. (b) apresenta $\rho_{2}$ de Sigma-g menos $0 \rho_{2}$ de DL.

$\rho_{1}$, marca-se o $\rho_{2}$ de Sigma-g menos o $\rho_{2}$ de DL. Essa figura traça as diferenças em $\rho_{2}$ para cada $\rho_{1}$ (Sigma-g menos DL). Sempre que a coordenada $y$ é positiva, Sigma-g tem melhor desempenho em termos de número de veículos que realizam contato durante o valor dado no eixo $x$. Quando o segmento de curva tem uma coordenada $y$ negativa, DL tem mais veículos que realizam conexões.

A linha azul compara Sigma-g e DL para uma Deposição $\Sigma\left(\begin{array}{c}R_{c} \\ c\end{array}\right)\left(\begin{array}{l}0.1 \\ 0.3\end{array}\right)$. Sigma-g supera DL quando a coordenada $x$ é maior do que 0,2. A seta azul (\#2) indica que Sigma-g apresenta $3 \%$ mais veículos que realizam contato durante $30 \%$ do tempo de duração da viagem. Da mesma forma, a linha verde indica uma Deposição $\Sigma\left(\begin{array}{c}R_{c} \\ c\end{array}\right)\left(\begin{array}{c}0.5 \\ 0.3\end{array}\right)$, e a seta verde (\#1) indica que Sigma-g apresenta $4 \%$ mais veículos que realizam contato durante $20 \%$ do tempo de duração da viagem. A curva ciano indica a Deposição $\Sigma\left(\begin{array}{c}R_{c} \\ c\end{array}\right)\left(\begin{array}{l}0.9 \\ 0.3\end{array}\right)$, e que Sigma-g apresenta $-4 \%$ veículos realizando conexões durante $70 \%$ da duração da viagem ${ }^{5}$.

\section{Conclusão}

Este trabalho apresenta uma investigação sobre o planejamento de redes veiculares para a distribuição de conteúdos. Propõe-se a Deposição Sigma como métrica para avaliar se uma determinada distribuição de unidades de comunicação é capaz de suportar a distribuição de conteúdos. Considera-se que a rede deve gerenciar a distribuição de diversos conteúdos que devem ser oferecidos para os veículos em locais específicos da malha viária sob qualidades de serviço distintas. Para resolver a Deposição Sigma, propõe-se uma heurística gulosa Sigma-g. Comparou-se a eficiência de Sigma-g com a estratégia intuitiva de implantar unidades de comunicação nos locais de maior tráfego da malha rodoviária. Os resultados demonstram que Sigma-g requer menos unidades de comunicação que DL para um mesmo patamar de qualidade de serviço ofertada.

\section{Agradecimentos}

Esse trabalho foi parcialmente financiado por CNPq, CAPES e FAPEMIG.

\footnotetext{
${ }^{5}$ Ressalta-se que esses resultados aplicam-se apenas ao rastro de mobilidade em análise.
} 


\section{Referências}

Bruno, F., Cesana, M., Gerla, M., Mauri, G., and Verticale, G. (2015). Optimal content placement in icn vehicular networks. In 5th IEEE International Conference on Network of the Future.

Cheng, H., Fei, X., Boukerche, A., and Almulla, M. (2015). Geocover: An efficient sparse coverage protocol for rsu deployment over urban vanets. Ad Hoc Networks, 24, Part B(0):85 - 102. Modeling and Performance Evaluation of Wireless Ad-Hoc Networks.

Gossa, J., Janecek, A. G., Hummel, K., Gansterer, W. N., Pierson, J.-M., et al. (2008). Proactive replica placement using mobility prediction. In Mobile Data Management Workshops, 2008. MDMW 2008. Ninth International Conference on, pages 182-189. IEEE.

Lee, J. and Kim, C. (2010). A roadside unit placement scheme for vehicular telematics networks. In Kim, T.-h. and Adeli, H., editors, Advances in Computer Science and Information Technology, volume 6059 of Lecture Notes in Computer Science, pages 196-202. Springer Berlin Heidelberg.

Li, Y., Jin, D., Hui, P., and Chen, S. (2015). Contact-aware data replication in roadside unit aided vehicular delay tolerant networks. Mobile Computing, IEEE Transactions on, $\mathrm{PP}(99): 1-1$.

Silva, C. M., Andre L. L. Aquino, and Wagner Meira Jr (2015a). "avaliando o desempenho de redes veiculares heterogêneas". In SBCUP 2015 (Simpósio Brasileiro de Computação Ubíqua).

Silva, C. M., Sergio Oliveira, Andre L. L. Aquino, and Fernando Augusto Teixeira (2013). PMCP: Uma Heurística Probabilística para Otimizar a Instalação de Pontos de Disseminação em Redes Veiculares. In SBCUP 2013 (Simpósio Brasileiro de Computação Ubíqua).

Silva, C. M., Wagner Meira Jr, and Joao F. M. Sarubbi (2015b). Non-Intrusive Planning of Roadside Infrastructure for Vehicular Networks Without Tracking Individual Vehicles. IEEE Transactions on Intelligent Transportation Systems, -(-):-.

Silva, F., Boukerche, A., Silva, T. R. M., Ruiz, L. B., Cerqueira, E., and Loureiro, A. A. (2014). Content replication and delivery in vehicular networks. In ACM International Symposium on Development and Analysis of Intelligent Vehicular Networks and Applications, DIVANet '14, pages 127-132, New York, NY, USA. ACM.

Teixeira, F., Silva, V., Leoni, J., Macedo, D., and Nogueira, J. M. S. (2014). "vehicular networks using the ieee 802.11p standard: An experimental analysis". Vehicular Communications, 1(2):91 - 96.

Trullols, O., Fiore, M., Casetti, C., Chiasserini, C., and Ordinas, J. B. (2010). Planning roadside infrastructure for information dissemination in intelligent transportation systems. Computer Communications, 33(4):432 - 442.

Xie, B., Xia, G., Chen, Y., and Xu, M. (2013). Roadside infrastructure placement for information dissemination in urban its based on a probabilistic model. In Network and Parallel Computing, volume 8147 of Lecture Notes in Computer Science, pages 322-331. Springer Berlin Heidelberg.

Zheng, Z., Lu, Z., Sinha, P., and Kumar, S. (2010). Maximizing the contact opportunity for vehicular internet access. In INFOCOM, 2010 Proceedings IEEE, pages 1-9. 